\title{
In-situ observation of movement of atoms and identification of thorium atoms by core-loss electron imaging
}

\author{
Y. Makita $\left({ }^{1}\right)$, H. Hashimoto $\left({ }^{1}\right)$, N. Nagaoka $\left({ }^{1}\right)$ and A. Kumao $\left({ }^{2}\right)$ \\ ( $\left.{ }^{1}\right)$ Faculty of Engineering, Okayama University of Science, Ridai-cho, Okayama 700, Japan \\ $\left({ }^{2}\right)$ Department of Electronics and Information Science, Kyoto Institute of Technology, Sakyo-ku, \\ Kyoto 606, Japan
}

(Received May 25, 1993 ; accepted June 21, 1993)

\begin{abstract}
In-situ observation of the process of coagulation and decomposition of long chain molecules of thorium-pyromeritate dispersed on graphite thin films and their crystallization process to small crystals of $\mathrm{ThO}_{2}$ under electron beam irradiation have been recorded by using the TV system which is located behind the fluorescent screen of JEOL $400 \mathrm{kV}$ electron microscope. Identification of thorium (Th) atoms is carried out using only the electrons having suffered an energy loss corresponding to the excitation of the $\mathrm{O}_{4,5}$ level in Th atoms.
\end{abstract}

\section{Introduction.}

The first transmission electron microscope images of Th atoms in Th-pyromeritate molecules and $\mathrm{ThO}_{2}$ small crystals were recorded in 1971 at $100 \mathrm{kV}$ by using dark field imaging method (Hashimoto et al., 1971)and not by bright field imaging method. This is due to the fact that the bright field image shows very small contrast. The observed images of Th atoms in molecules and crystals showed the linear arrangement and crystalline structure of bright spots of $0.3 \mathrm{~nm}$ in diameter respectively. Their arrangements agreed with the ones of individual atom in molecules and crystals and not just like lumps which were observed by scanning electron microscopes about one year before (Crewe et al., 1970). Even if the dark field images of atoms have high contrast, the intensity is very weak $\left(2 \times 10^{-} 3\right)$ and thus, for recording the moving atoms by using TV system, the intensity of illuminating electron beam was increased to be $100 \mathrm{amp} / \mathrm{cm}^{2}$ and $1 / 3 \sim 1 / 4 \mathrm{sec}$ onds accumulation of the images on the target of TV camera was carried out before displaying on the TV screen and recording on the video tape. The displayed or recorded images, appearing intermittently on the display monitor or on the tape, were recorded in $16 \mathrm{~mm}$ movie films continuously by driving the movie camera with a pulse motor which is synchronized to the intermittently recorded images. Since $16 \mathrm{~mm}$ movie films were then projected at a speed of 24 frames per second, the speed of the moving atoms was raised by a factor of 6 to 8 times (Hashimoto et al., 1978 - 79). 
In the present work, illuminating electrons are $400 \mathrm{kV}$ electrons and thus the intensity of the scattered electrons is 2.2 times larger than in $100 \mathrm{kV}$ in consequence of the relativistic effect of electron mass and the brightness of electron gun which is function of the accelerating voltage is also increased compared with $100 \mathrm{kV}$ electrons. Furthermore, the resolution limit of the $400 \mathrm{kV}$ electron microscope is $0.18 \mathrm{~nm}$, which is higher than $0.3 \mathrm{~nm}$ in the $100 \mathrm{kV}$ electron microscope. These conditions enable to observe the images of Th atoms with high contrast, even in the bright field imaging mode without accumulation of imaging electrons, and to record the movement of atoms by decomposition and crystallization process.

The conventional method to identify the kind of atoms from their electron microscope images is to compare the observed image contrat with the calculated one taking account the electron scattering process by the atoms of the target and the image formation process by the electron lenses (Hashimoto et al., 1973, 1978, Stadelman, 1987). This method, however, is an indirect method for identifying atoms and thus a direct method is preferable. The characteristic energy loss spectrum of the electrons passing through the specimen contains the most reliable and useful information for this purpose. In the present paper, images of atoms formed by using only the characteristic energy loss electrons (i.e. electrons having excited core electrons in atoms) can be used for the identification of Th atoms (Hashimoto et al., 1992, 1993).

\section{Experimental.}

Th-pyromeritate was prepared by the reaction of thorium nitride and pyromeritic acid and has the chemical structure of a long chain molecule containing several Th atoms separated by a distance of about one $\mathrm{nm}$. The specimen was prepared by putting a drop of water of $10^{-5}$ mol solution on a micro-grid supporting cleaved thin natural graphite films and was mounted in the top entry goniometer of JEOL $400 \mathrm{kV}$ electron microscope $\left(\mathrm{LaB}_{6}\right.$ filament gun) with the resolution limit of $0.18 \mathrm{~nm}$ which equipped a objective pole piece of spherical aberration coefficient $C_{\mathrm{s}}=1.0 \mathrm{~mm}$ and chromatic aberration coefficient $C_{\mathrm{c}}=1.9 \mathrm{~mm}$.

The movement of atoms due to the decomposition and recrystallization was recorded directly by a TV camera, which is positioned just behind the fluorescent viewing screen, and by normal photographic films.

Characteristic Th atoms images were formed by selecting the thorium $\mathrm{O}_{4,5}$ loss electrons with a slit in the spectrum plane at the back of the magnetic sector, and recording them on a slow scan CCD camera (Krivanek et al., 1993) without any distortion due to the six quadrupole lenses and six sextupole lenses of the projection system (Hashimoto, 1980, Krivanek et al., 1992a,b). Similarly electron diffraction patterns on the viewing fluorescent screen could be projected on the slow scan CCD camera by using only the characteristic loss electrons. The energy filtered images and patterns were then displayed and printed using a Macintosh computer.

\section{Observation.}

The specimen Th-pyromeritate on graphite supporting film is decomposed under $400 \mathrm{kV}$ electron beam irradiation and becomes $\mathrm{ThO}_{2}$ crystals after several minutes of irradiation. Long chain molecules are changed to small crystals of $\mathrm{ThO}_{2}$ as shown in figure 1 , which is confirmed by the selected area electron diffraction pattern. The arrows $\mathrm{A}, \mathrm{B}$ and $\mathrm{C}$ show small crystals with sizes of about one, two and seven unit cells of $\mathrm{ThO}_{2}$ crystals. The strings shown by arrows $\mathrm{D}$ and $\mathrm{E}$ are likely images of chain molecules containing Th atoms. The process of decomposition and crystallization has been recorded in VTR in real time. Vivid movement of atoms during the formation 
of small crystals has been observed on TV screen. It is rather difficult to reproduce such vivid movement by static series of photographs, but some sequences of the frames of TV images (1/30 seconds intervals) are duplicated in figures $2(\mathrm{a}-\mathrm{j})$. Small displacement of atoms was revealed by making the difference of successive frames using the subtraction technique (Hashimoto et al., 1991). Quantitative fluctuation of atom images during the crystal growth will be shown elsewhere, but by referring to the arrows, which show the same area of the specimen, some changes are seen. At the initial stage of illumination rather uniform strings are seen. Before crystallization they become spotty strings and change to crystals. This may be due to the evaporation of carbon, oxygen and hydrogen atoms when the decomposition of Th-pyromeritate molecules is taking place.

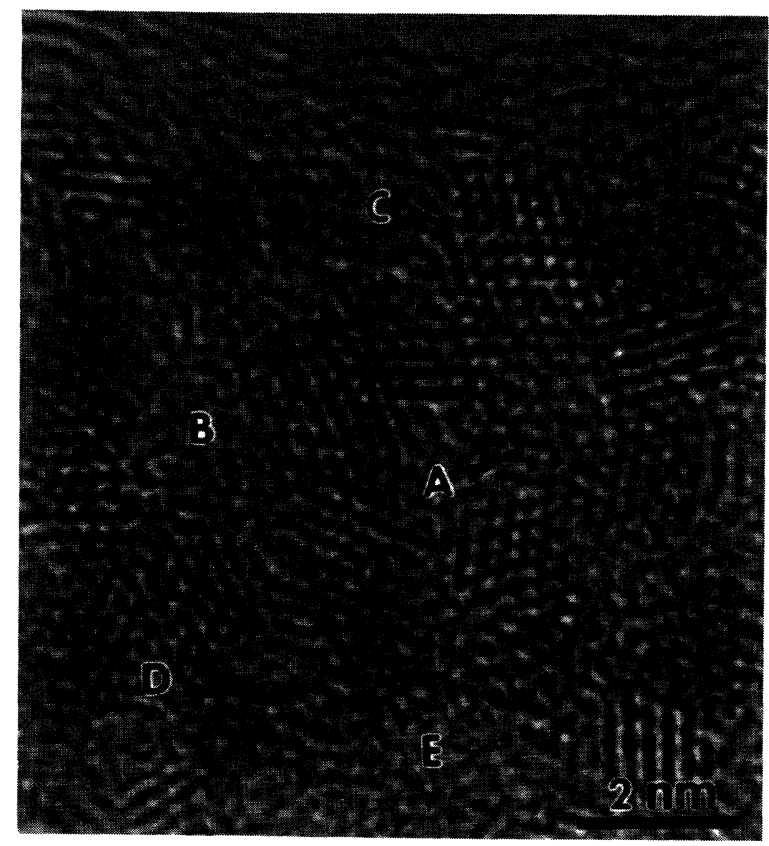

Fig. 1. - Bright field image of Th-pyromeritate and $\mathrm{ThO}_{2}$ crystals formed by the irradiation with $400 \mathrm{kV}$ electrons on a graphite thin film. $\mathrm{A}, \mathrm{B}$, and $\mathrm{C}$ show the $\mathrm{ThO}_{2}$ crystals of about one, two and seven unit cells. $\mathrm{D}$ and $\mathrm{E}$ show the strings with weak spotty contrast, which may be Th-pyromeritate molecules.

In order to identify the existence of Th and $\mathrm{O}$ atoms in crystallized structure, energy spectrum and energy selected (filtered) electron micrographs have been acquired. Figure 3 is an energy loss spectrum of thorium oxide crystals and Th-pyromeritates supported on graphite as shown in figure 1. The plasma loss peak $(24 \mathrm{eV}), \mathrm{O}_{4,5}$ peak of thorium atoms $(98 \mathrm{eV})$ and $\mathrm{K}$ peak of oxygen atoms $(530 \mathrm{eV})$ are seen similarly to previous observations at $200 \mathrm{kV}$ (Hashimoto et al., 1992, Krivanek et al., 1992a,b).

Figures $4 \mathrm{a}, \mathrm{b}$ and $\mathrm{c}$ are the images of $\mathrm{ThO}_{2}$ crystals and Th-pyromeritate molecules on a graphite supporting film, using no loss electrons, $98 \mathrm{eV}$ energy loss electrons (thorium $\mathrm{O}_{4,5}$ ) and $530 \mathrm{eV}$ energy loss electrons (oxygen $\mathrm{K}$ ), with an energy window of $5 \mathrm{eV}$. Since the intensities of these peaks are very different, the exposures were $0.3 \mathrm{~s}, 3 \mathrm{~s}$ and $10 \mathrm{~s}$ for (a), (b) and (c) respectively. Since the intensity of energy loss electrons from oxygen atoms is about 2000 times smaller than that of zero loss electrons, $10 \mathrm{~s}$ exposures are not long enough to record clearly the images of oxy- 
a)

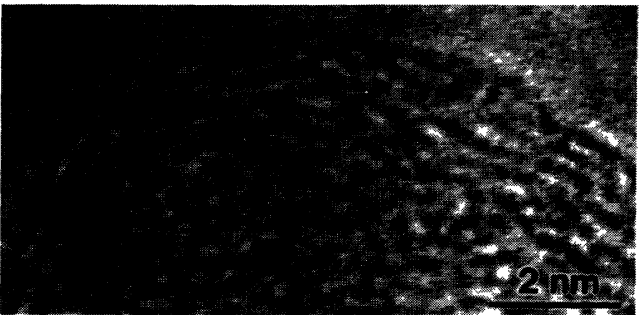

b)

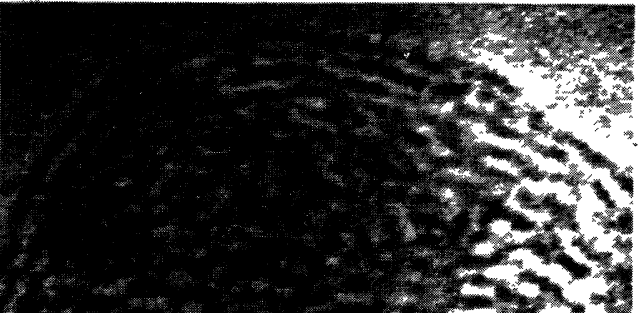

c)

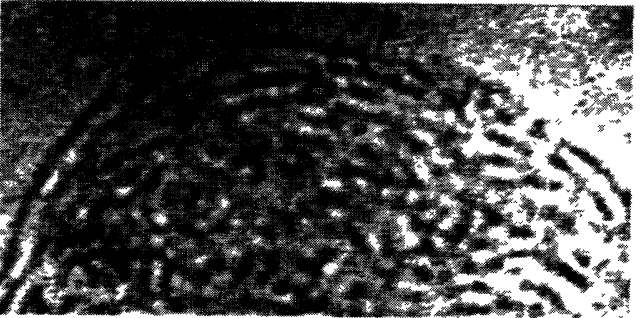

d)
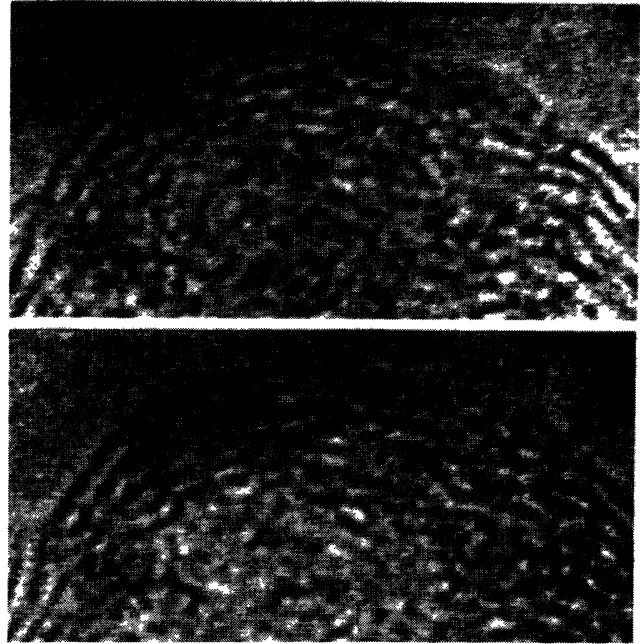
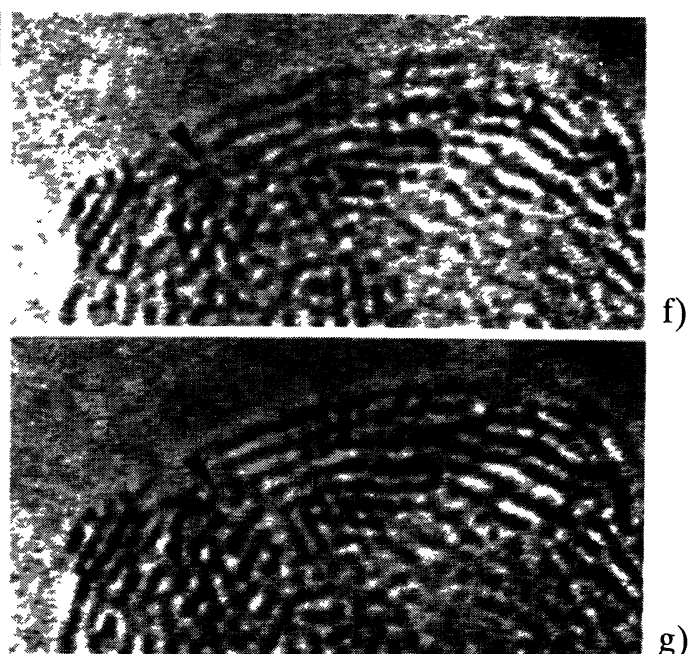

g)

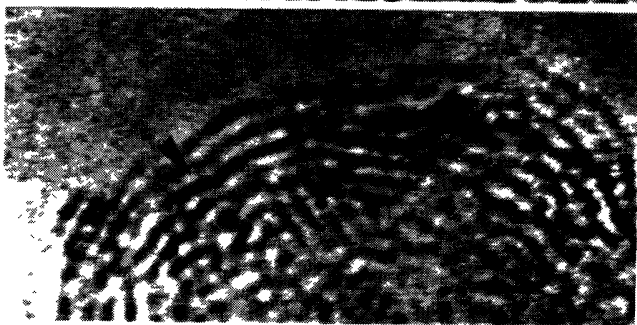

h)

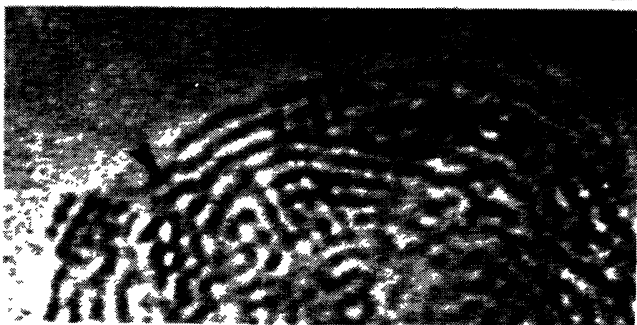

i)

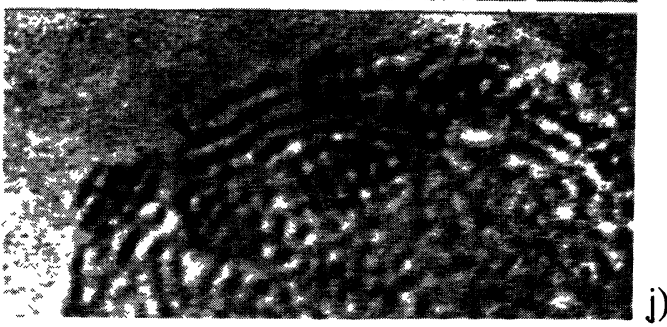

Fig. 2. - Growing process of $\mathrm{ThO}_{2}$ crystals by $400 \mathrm{kV}$ electron beam irradiation. Th-pyromeritate chain molecules change to $\mathrm{ThO}_{2}$ crystals (a-e) and smooth chains change to spotty chain (f-j). Intervals are (in seconds)(a)-0.4-(b)-0.4-(c)-0.4-(d)-0.4-(e)-464.0-(f)-0.4-(g)-0.2-(h) -1.0-(i)-2.4-(j).

gen atoms. However much longer exposure time produce drift-blurred image, as the drift should be less than $0.05 \mathrm{~nm}$ to obtain atomic resolved images.

Atomic resolved images and corresponding diffraction patterns were observed using zero loss, plasmon loss and core loss electrons of crystals with different thickness. Figures $5 \mathrm{a}$ and $\mathrm{b}$ are the 


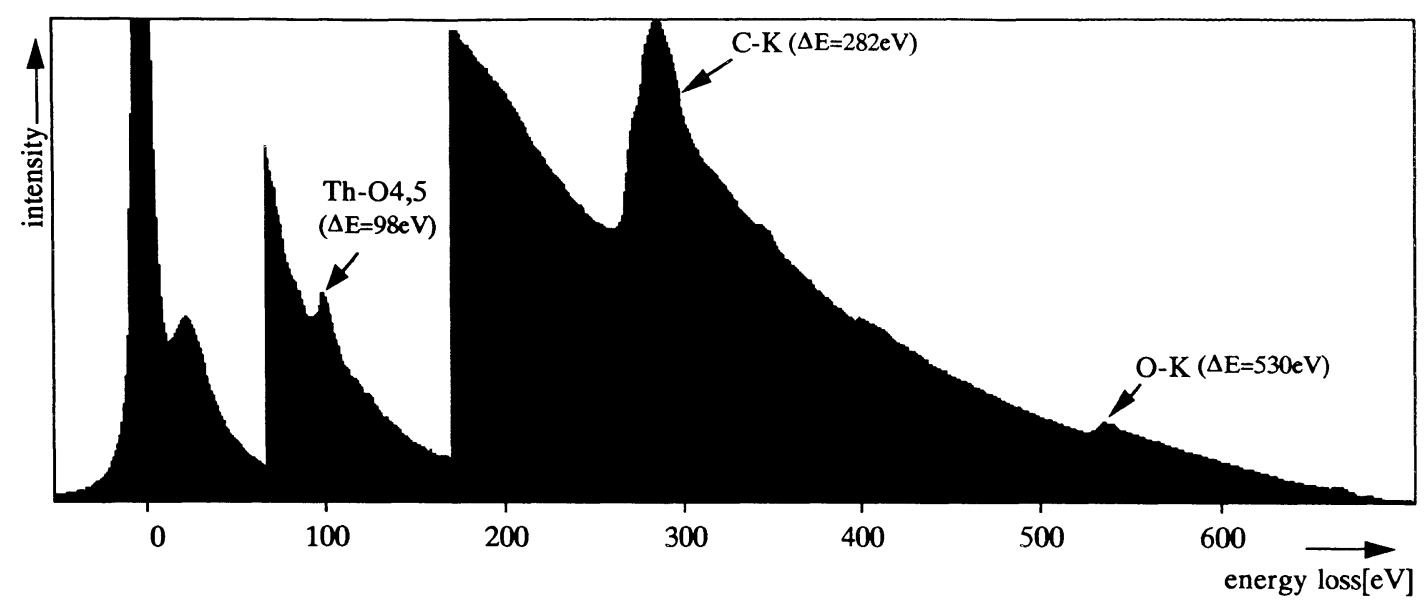

Fig. 3. - Energy loss spectrum from the specimen of $\mathrm{ThO}_{2}$ and Th-pyromeritate.

zero loss and $98 \mathrm{eV}$ energy loss images of $\mathrm{ThO}_{2}$ crystals without the supporting graphite film. Some more electron microscope images were also photographed by setting the energy selecting window to the lower and higher energy positions than $98 \mathrm{eV}$. In these scatterings, though the intensity of illuminating electron beams was increased enough to detect the atomic resolved images, the image at lower energy position are hardly seen but at higher energy position can be seen even they are low contrast. This observation seems to suggest that the detectability of atomic resolved images is not in the intensity but the nature of the $98 \mathrm{eV}$ peak. Diffraction patterns using the zero loss and $98 \mathrm{eV}$ loss electrons are also shown for thin and thick crystals in figures 6 (a, b) and (c, d) respectively. The diffraction patterns in figure 6 showed high contrast in (a, c) and only strong diffuse central spot with no diffraction rings in (b) and very weak diffuse rings in (d). Very weak diffuse diffraction rings appear only when the size of specimen crystals is larger than about several nanometers. The broadening of diffraction pattern in figure $6 \mathrm{a}$ seems to be due to the specimen crystal size and illuminating beam divergence. Since it is rather difficult to take the electron diffraction pattern corresponding to the small area shown in figure $5 \mathrm{a}$, the patterns in figure 6 have ring structure.

\section{Discussion and conclusion.}

In order to characterize the atoms in $\mathrm{ThO}_{2}$ crystals, it seems necessary to discuss the formation process of the images by energy filter. However, since accurate interpretation of inelastic image could be made by collecting some more observations, we discuss only briefly here.

After the experiment of Kamiya and Uyeda (1961) suggesting that the diffraction contrast or interference effects observed in the electron microscope images of crystals are not only due to the elastically scattered electrons but also by the inelastically scattered electrons, a number of authors (Fujimoto, Kainuma, Howie, Fukuhara) independently developed theories of small angle inelastic scattering in crystals in around 1961-1963. In 1974, (Hashimoto, 1974) it was demonstrated that the images of bend contours formed by the inelastic-elastic scattered electrons (Kikuchi electrons) and the elastic-inelastic scattered electrons (anti-Kikuchi electrons) can be recorded separately in an image of bent crystal. The quantum mechanical discussion based on the plasmon scattering was also shown (Hashimoto and Kumao, 1988). 

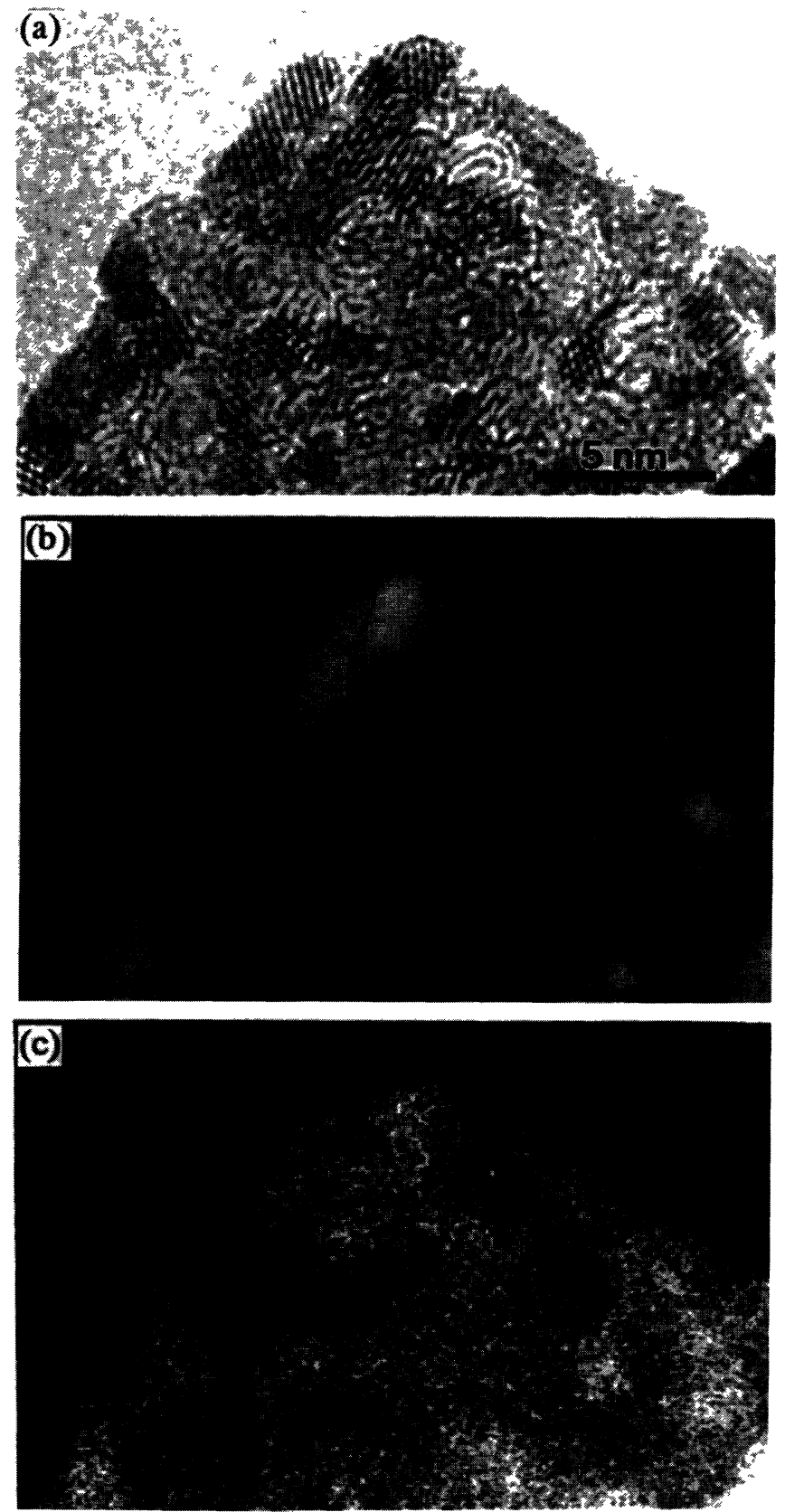

Fig. 4. - Energy filtered images of $\mathrm{ThO}_{2}$ and Th-pyromeritate on graphite substrate. a) zero loss image, b) image formed by $98 \mathrm{eV}$ energy loss electrons (characteristic loss of $\mathrm{O}_{4,5}$ of Th atoms), c) $530 \mathrm{eV}$ energy loss electrons (characteristic loss of image by $\mathrm{K}$ level of oxygen).

The crystal lattice fringe formed by using only plasmon loss spectrum was recorded in 1977 using STEM (Craven et al., 1977). The atomic structure image using plasmon loss spectrum was demonstrated together with the theoretical contrast calculation in 1985 (Ajika et al., 1985). Since 

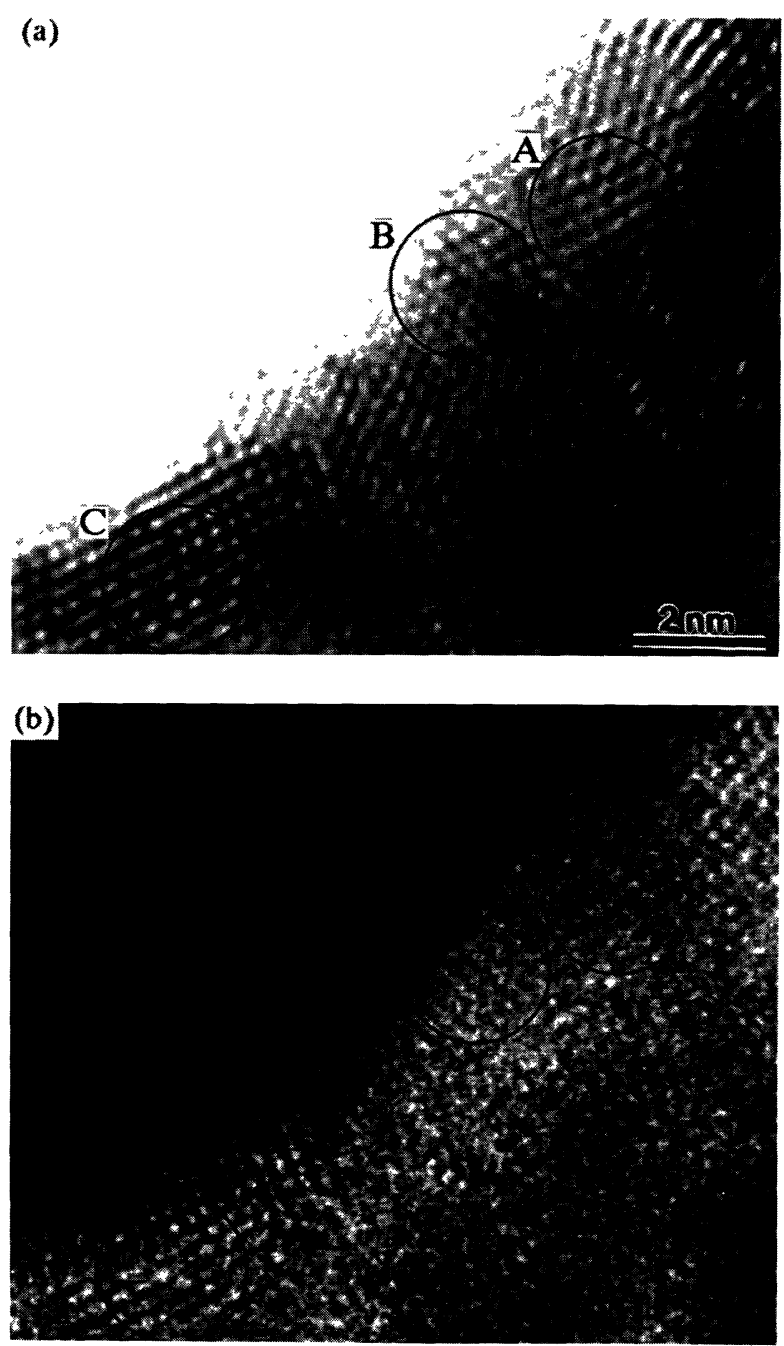

Fig. 5. - Atom images of $\mathrm{ThO}_{2}$ crystals without supporting film. a) zero loss image, b) image by $98 \mathrm{eV}$ energy loss electrons $\left(\mathrm{O}_{4,5}\right.$ of $\mathrm{Th}$ atoms). See also the image contrast difference of $\mathrm{a}$ ) and $\mathrm{b}$ ), in the regions indicated by $\mathrm{A}, \mathrm{B}$ and $\mathrm{C}$.

the image had a different magnification in the $X$ and the $Y$ direction, the images were heavily distorted which was corrected by a computer after recording. In 1986, (Krivanek et al., 1986) the atomic structure image without first order aberration and distortion was obtained using plasma loss electrons. In 1992, Krivanek et al. (Krivanek et al., 1992a) and Hashimoto et al. (Hashimoto et al., 1992) observed the atomic structure images of $\mathrm{Th}$ atoms in $\mathrm{ThO}_{2}$ crystals using core loss electrons in the $200 \mathrm{kV}$ electron microscope fabricated with newly developed Gatan imaging filter. Krivanek et al. (Krivanek et al., 1992b) discussed the energy loss dependence of the contrast of atomic resolution image and the intensity of diffraction pattern.

The energy filtered electron microscope images and diffraction patterns which are formed by 

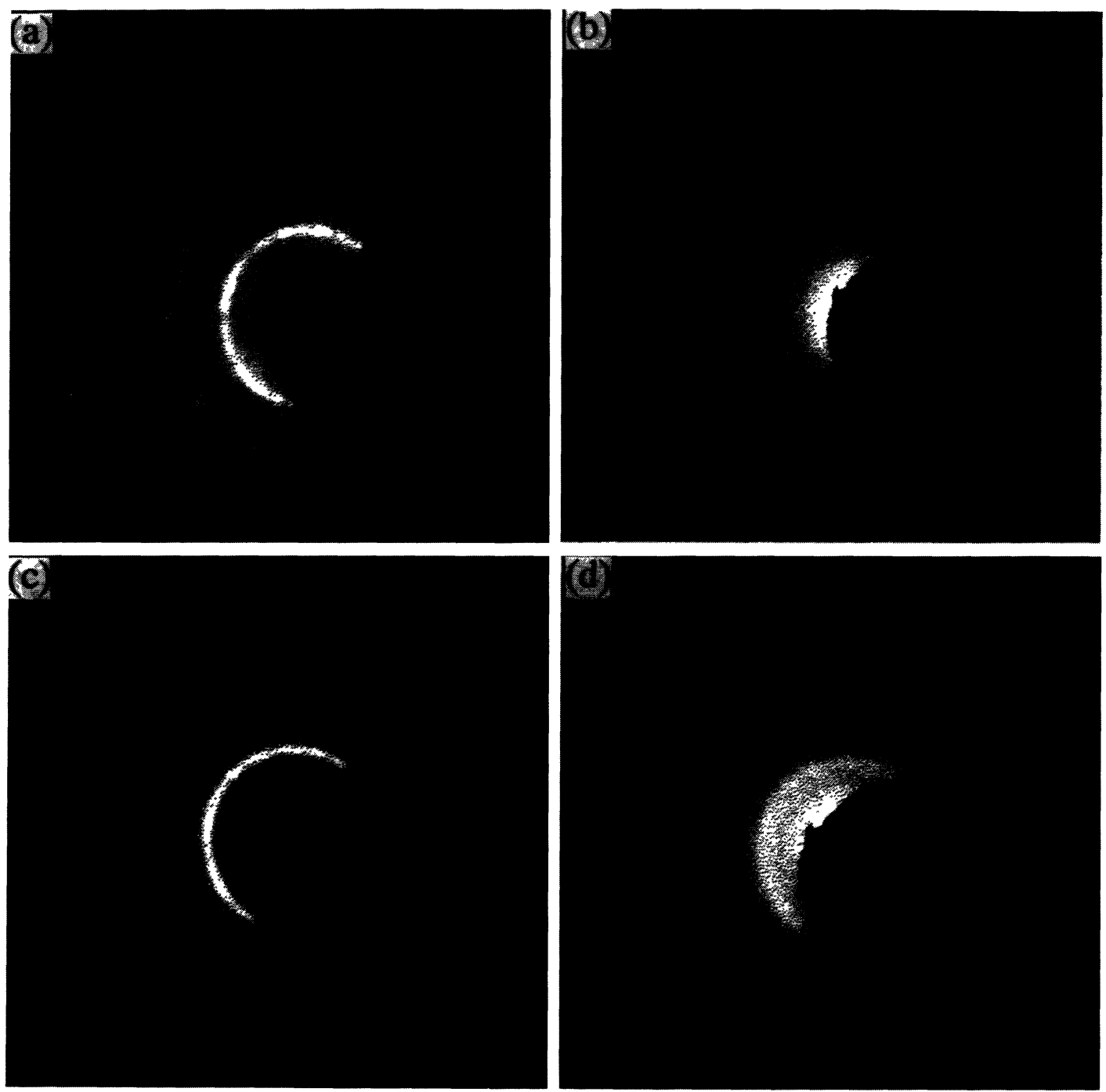

Fig. 6. - Electron diffraction patterns of $\mathrm{ThO}_{2}$ crystals supported on garphite thin films. (a, c) zero loss electron diffraction pattern, (b, d) $98 \mathrm{eV}$ loss electron diffraction pattern. (a, b) are from thin region (less than $3 \mathrm{~nm}$ ), (c, d) thick region.

characteristic loss electrons are produced by the various combination of successive elastic and inelastic scattering (Spence et al., 1982). As the mean free path and the probability of scattering are different in elastic scattering and inelastic scattering, the image contrast and intensity of diffraction pattern change with specimen thickness. Existence of the elastic scattering process in the inelastic scattering can well be detected in the energy filtered electron diffraction patterns. If the elastic scattering process is taken place before or/and after an inelastic scattering, diffraction rings will appear, whose intensity is proportional to the number of elastic scatterings in the kinematic scattering process. Thus the detectability of diffraction rings can be the measure of existence of the amount of existence of elastic process in the scattering. No observable diffraction rings 
in figure $6 \mathrm{~b}$ suggest that hardly detectable elastic scatterings were taken place before and after the inelastic scattering. In this specimen, however, elastic scatterings without combining inelastic scattering were taken place as can be seen in figure $6 \mathrm{a}$. This observation seems to suggest that the energy filtered atomic images appearing in thin region of thorium oxide using $98 \mathrm{eV}$ shown in figure $5 \mathrm{~b}$ are the images of Th atoms. This observation seems to agree with STEM observations (Mory et al., 1989, Krivanek et al., 1991a,b). By comparing figures 5a and b, it is seen that atomic structure image is slightly different as seen in the regions $\mathrm{A}, \mathrm{B}$ and $\mathrm{C}$. In the regions $\mathrm{A}, \mathrm{B}$ and $\mathrm{C}$, two cross fringes are predominant in (a) but only one directional arrangements of bright spots are visible. These difference is not due to the change of specimen structure, orientation and lens focusing. This was confirmed by the image taken by zero loss electrons immediately after recording the image of (b). The cross fringes in the zero loss images are seen to be due to the superposition of two small crystals. Inelastic dotted images are arranged parallel to the fringes of one of the crystals, i.e. top or bottom crystals superposed. This phenomena will give us an information on the formation process of energy filtered images.

In conclusion, the present observations using a TV system and energy filtering device have demonstrated the existence of Th atoms and their slight movements accompanied by the growth of $\mathrm{ThO}_{2}$ small crystals under the $400 \mathrm{kV}$ electron beam irradiation.

\section{Acknowledgements.}

The authors would like to acknowledge Professors Kamiya and Ichinokawa and also Dr. Krivanek of Gatan Inc. and the referees of the present paper for helpful discussions and comments and the support of Okayama University of Science, the Ministry of Education and Culture, and the helps of JEOL Co. and Gatan Inc. They are also indebted to Prof. Yokota of Okayama University of Science and Dr. Ishida of JEOL for their helps for installing and operating the $400 \mathrm{kV}$ energy filtering microscope.

\section{References}

[1] Ajika N., Hashimoto H., Yamaguchi K. and Endoh H., Jpn. J. Appl. Phys. 24 (1985) L41.

[2] Craven A.J. and CollieX C., J. Microsc. Spectorsc. Electron 2 (1977) 511.

[3] Crewe A.V., WALl J. and LANGMORE J., Science 168 (1970) 1338.

[4] Hashimoto H., Kumao A., Hino K., Yotsumoto H. and Ono A., Jpn. J. Appl. Phys. 10 (1971) 1115.

[5] Hashimoto H., KumaO A., Hino K., ENDOH H., Yotsumoto H. and ONO A., J. Electron Microsc. 22 (1973) 123.

[6] HaShimoto H., Proc. 3rd Int. Nat. Conf. HVREM Oxford, Academic Press 7 (1974).

[7] Hashimoto H., KumaO A. and Endoh H., Electron Microsc. (Toronto) 3 (1978) 244.

[8] Hashimoto H., Yokota Y., Takai Y., Endoh H. and Kumao A., Chemica Scripta, (Proc. Novel Symp.) 14 (1978-79) 125.

[9] HaShimoto H., Jpn. Patent. S62-37503 (1980).

[10] Hashimoto H. and Kumao A., Phys. Stat. Sol. (a) 107 (1988) 611.

[11] Hashimoto H., MaKita Y., Yokota Y., IKUTA T., HaShimoto M. and Hetherington C.J.D., Ultramicroscopy 39 (1991) 171.

[12] Hashimoto H., MakiTa Y. and NAGaOKa N., Proc. EMSA Boston 2 (1992) 1194.

[13] HaShimoto H., Makita Y., NAGAOKa N. and Yokota Y., Optik (Memorial issue of Prof. Möllenstedt, 1993) in press.

[14] KamiYa Y. and UYeda R., J. Phys. Soc. Jap. 16 (1961) 1361.

[15] KRIVANEK O.L. and AHN C.C., Electron Microsc. (Kyoto Congress) 1 (1986) 519.

[16] KrivaneK O.L., Mory C., Tence M. and Colliex C., Microsc. Microanal. Microstr. 2 (1991) 257. 
[17] KrivaneK O.L., Gubbens A.J. and Dellby N., Microsc. Microanal. Microstr. 2 (1991) 315.

[18] Krivanek O.L., Gubbens A.J., Dellby N. and Meyer C.E., Proc. EMSA Boston 2 (1992a) 1192.

[19] KrivaneK O.L., Gubbens A.J., Dellby N. and Meyer C.E., Microsc. Microanal. Microstr. 3 (1992b) 187.

[20] KRIVANEK O.L. and MoONEY P.E., Ultramicroscopy 49 (1993) 95.

[21] Mory C. and COllieX C., Ultramicroscopy 28 (1989) 339.

[22] SPENCE J.C.H. and LYNCH J., Ultramicroscopy 9 (1982) 267.

[23] STADELMAN P., Ultramicroscopy 21 (1987) 131. 